\title{
Chemical Shift Misregistration Effect in Spinal Magnetic Resonance Imaging
}

\author{
Masaki KomiYama, Toshihiro Yasui, Hisatsugu Yagura, Yoshihiko FU, \\ Hajime SugATA, Miki KIYAMA and Mitsuru BABA
}

Department of Neurosurgery, Baba Memorial Hospital, Sakai, Osaka

\begin{abstract}
The purpose of this study was to identify the manifestations of the chemical shift misregistration effect in spinal magnetic resonance (MR) images. With the use of a 0.5 -T superconducting MR scanner, axial images of a normal lumbar spine were obtained with $T_{1}$-weighted spin echo sequences in which the frequency- and phase-encoding gradients were interchanged. A low intensity meniscus (band) was demonstrated around the thecal sac in the direction of the lower frequency-encoding gradient. Awareness of this phenomenon is crucial to the correct interpretation of spinal MR images, especially in cases of lipoma or other fatty tumors.
\end{abstract}

Key words: chemical shift, magnetic resonance imaging, spinal cord lesions

\section{Introduction}

The chemical shift misregistration effect in magnetic resonance (MR) images has been well documented. ${ }^{1,4)}$ It is usually seen at the junction of fatty and nonfatty tissues - for example, where the optic nerve enters the orbit and where the perirenal fat abuts the renal parenchyma. We studied this phenomenon in spinal MR images so as to avoid misinterpretation, and we report and discuss our findings.

\section{Materials and Methods}

The MR scanner employed was a 0.5-T superconducting system (Picker International, Cleveland, $\mathrm{OH}$ ). The pulse sequences were $T_{1}$-weighted spin echo (SE) with repetition (TR) and echo (TE) times of 600 and $40 \mathrm{msec}$, respectively. Image reconstruction was performed by a two-dimensional Fourier transformation method. A body coil was used as a transmitting antenna and a surface coil for the lumbar spine as a receiving antenna. Slice thickness was approximately $1.0 \mathrm{~cm}$ and the field diameter $30 \mathrm{~cm}$. The data matrix was $256 \times 256$; the data were averaged twice. Each pixel was about $1.2 \times 1.2 \mathrm{~mm}$. The strength of the frequency-encoding gradient was $0.985 \mathrm{mT} / \mathrm{m}$.

Received December 5, 1986; Accepted February 15, 1988
Thus, the band width for each pixel in our system was about $50 \mathrm{~Hz}$. Axial images were obtained.

Our first subject was a 26-year-old male volunteer with no clinical or radiological evidence of a lumbar lesion. The frequency-encoding (read-out) gradient was vertical from the subject's posterior to anterior, and the phase-encoding gradient, which was perpendicular to the frequency-encoding gradient, was horizontal from his right to left. In obtaining the second image we reversed the encoding gradients; that is, with the frequency-encoding gradient from his right to left and the phase-encoding gradient from his posterior to anterior, we imaged exactly the same plane. We also examined a 16-year-old male with a thoracic spinal cord lipoma, using the same pulse sequences. In this case, the frequency-encoding gradient was vertical from his posterior to anterior. The chemical shift misregistration effect was evaluated in both cases.

\section{Results}

In the normal subject's lumbar image, the thecal sac was surrounded by the epidural fatty tissue, which was prominent at the dorsal aspect of the spinal cord. A low intensity meniscus (band) was noted mainly in the dorsal aspect of the thecal sac on the image with the vertical frequency-encoding gradient from posterior to anterior (Fig. 1 left). On the other 


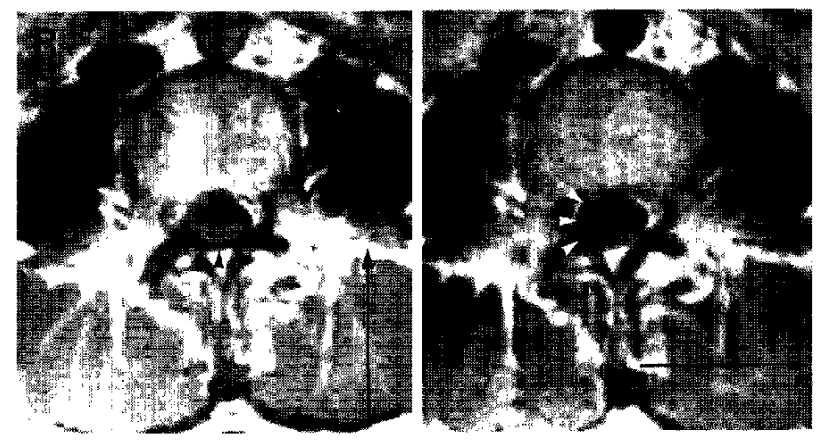

Fig. $1 \mathrm{~T}_{1}$-weighted $\mathrm{SE}$ images ( $\mathrm{TR} / \mathrm{TE}, 600 / 40 \mathrm{msec}$ ) of a normal lumbar spine. The arrow indicates the direction of the frequency-encoding (readout) gradient. left: A low intensity meniscus (arrowheads) is seen at the dorsal aspect of the thecal sac. right: A low intensity meniscus (arrowheads) is visible at the right lateral aspect of the thecal sac.

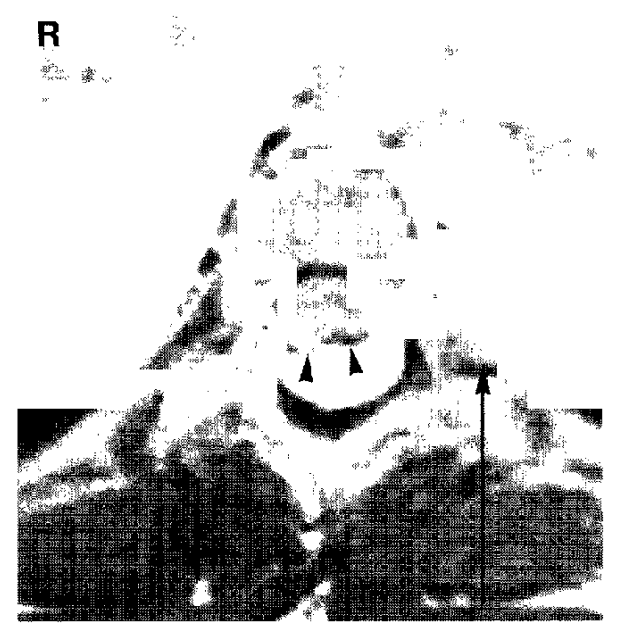

Fig. $2 \mathrm{~T}_{1}$-weighted SE image (TR/TE, 600/40 msec) of a thoracic lipoma. Note the low intensity band (arrowheads) between the spinal cord and the tumor. The tumor is intradural, extramedullary, and infiltrates and is firmly attached to the spinal cord. The arrow indicates the direction of the frequency-encoding gradient.

hand, this low intensity meniscus lay mainly in the right lateral aspect of the thecal sac on the image with the right-to-left horizontal frequency-encoding gradient (Fig. 1 right). The high intensity bands opposite these low intensity menisci in the above-described images were difficult to see, primarily because of the high intensity of the epidural fat.

In the case of the spinal cord tumor, a low inten- sity band was observed between the spinal cord and the tumor (Fig. 2). The high intensity tumor was situated in the dorsal portion of the spinal cord. The spinal cord and tumor were surrounded by a low intensity circle, which represented the cortex of the vertebral body. The epidural fat surrounding the thecal sac in the normal region was not observed. The operative findings were that the tumor was intradural and extramedullary at the dorsal aspect of the spinal cord. It was firmly attached to, and infiltrated, the spinal cord. The histopathological diagnosis was lipoma.

\section{Discussion}

Chemical shift occurs because the protons in fatty and nonfatty tissues resonate at slightly different frequencies, which is attributed to differences in their chemical environments. Protons in water precess slightly faster than those in fat. The chemical shift separation of the fat and water protons is about 3.03.5 parts per million. ${ }^{4)}$ At $0.5 \mathrm{~T}, 21 \mathrm{MHz}$ of Larmor frequency, the separation frequency is about 63$73.5 \mathrm{~Hz}$. Since the band width for a pixel in our MR system is about $50 \mathrm{~Hz}$, the "fat" image shifts about $1.6-1.8 \mathrm{~mm}$ (1.3-1.5 pixel) with respect to the "water" image. In MR imaging, this phenomenon occurs in the frequency-encoding direction, whereas phase encoding is not affected. This is because locational information in the direction of the phase-encoding gradient is derived from the signal phase, not from its frequency. Chemical shift is directly proportional to the main magnet strength and inversely proportional to the gradient field strength.

Protons in fatty tissue resonate at a lower frequency than those in nonfatty tissue. This means that the "fat" image shifts, with respect to the "water" image, in the direction of the lower frequency gradient. In spinal MR imaging, the spinal cord and cerebrospinal fluid give the "water" image, while the epidural fat provides the "fat" image. Due to the different chemical shifts of the "fat" and "water" images, pixel misregistration occurs, producing an artificial low intensity area. Figure 3 diagrammatically illustrates this phenomenon. The "fat" image shifts in direction of the lower frequency-encoding gradient. Thus, the low intensity meniscus around the thecal sac is produced in this direction.

The lipoma described above was initially diagnosed as an extradural tumor because the low intensity band was interpreted as the dura mater and the lack of epidural fat was also misleading. Usually, epidural fat is scant or absent when a spinal cord 

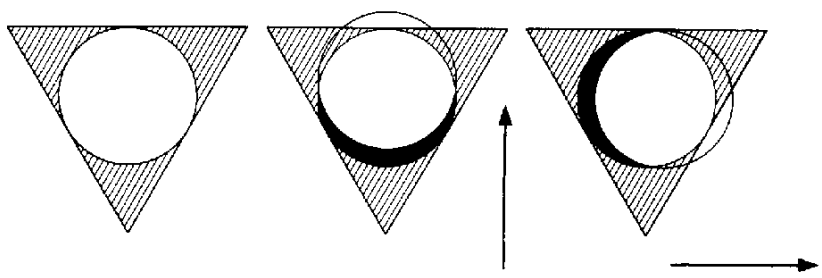

Fig. 3 Schematic illustrations of the chemical shift misregistration effect on spinal MR images. The arrow indicates the direction of the frequency-encoding gradient. left: The circle represents the thecal sac and the shaded triangle the epidural fat around the thecal sac. center: The epidural fat image shifts in the direction of the lower frequency-encoding gradient. A low intensity meniscus is seen at the dorsal aspect of the thecal sac. right: A low intensity meniscus is observed at the left lateral aspect of the thecal sac.

tumor is present. Hashimoto et al. ${ }^{3)}$ reported that the low intensity band between an extradural tumor and the spinal cord appears to represent the dura mater, and this knowledge is helpful in differentiating extradural from intradural, extramedullary tumors. However, it is not applicable in cases of lipoma or other fatty tumors because a similar low intensity band is produced by the chemical shift misregistration effect. To minimize this effect in the orbit, Daniels et al. " positioned the patient so that the optic nerve was parallel to the frequency-encoding gradient. Another solution, reported by Dixon, ${ }^{2}$ ) is to combine the "fat" and "water" images by the use of a computer. For spinal MR images, it is impossible to minimize chemical shift through positioning, since the spinal cord is round in axial images. Artificial combination of the "fat" and "water" images is not yet technologically possible, but in the future it will play an important role in eliminating the chemical shift misregistration effect. For the present, it is of vital importance to understand this phenomenon, so that spinal MR images are not misinterpreted.

\section{References}

1) Daniels DL, Kneeland JB, Shimakawa A, Pojunas KW, Schenck JF, Hart H, Foster T, Williams AL, Haughton VN: MR imaging of the optic nerve and sheath: Correcting the chemical shift misregistration effect. $A J N R$ 7: 249-253, 1986

2) Dixon WT: Simple proton spectroscopic imaging. Radiology 153: 189-194, 1984

3) Hashimoto H, Matsumura Y, Shakudo M, Takemoto $K$, Fukuda $T$, Inoue $Y$, Onoyama $Y$, Hakuba $A$, Yagura H, Baba M: MR imaging of intraspinal tumor. New extradural sign. Nippon Igaku Hoshasen Gakkai Zasshi 46: 500-502, 1986 (in Japanese)

4) Soila KP, Viamonte MV, Starewicz PM: Chemical shift misregistration effect in magnetic resonance imaging. Radiology 153: 819-820, 1984

Address reprint requests to: M. Komiyama, M.D., Department of Neurosurgery, Baba Memorial Hospital, 244 Higashi-4, Hamadera-funao-cho, Sakai, Osaka 592, Japan. 